\title{
Vibrio pacinii sp. nov., from cultured aquatic organisms
}

Correspondence

B. Gomez-Gil

bruno@victoria.ciad.mx

\author{
B. Gomez-Gil,, ${ }^{1}$ F. L. Thompson,,3 C. C. Thompson ${ }^{2}$ and J. Swings ${ }^{2,3}$ \\ 'CIAD/Mazatlán Unit for Aquaculture, AP 711, Mazatlán Sinaloa, México 82000 \\ ${ }^{2}$ Laboratory for Microbiology, Ghent University, Ghent 9000, Belgium \\ ${ }^{3}$ BCCM/LMG Bacteria Collection, Laboratory for Microbiology, Ghent University, Ghent 9000, \\ Belgium
}

\begin{abstract}
Three strains were isolated from cultured aquatic organisms. They were Gram-negative, oxidase-positive, motile, fermentative, arginine dihydrolase-positive, lysine and ornithine decarboxylase-negative and sensitive to vibriostatic agent O/129. These strains differ from other related Vibrio species by several phenotypic features, which include acetoin and indole production and utilization of amygdalin and D-mannitol. Comparison of $16 \mathrm{~S}$ rDNA sequences showed a close relationship to the recently described species Vibrio kanaloae $(96.6 \%)$ and Vibrio pomeroyi (96.4\%) and to Vibrio furnissii (96.6\%), but DNA-DNA hybridization experiments showed that the three isolates form a tight novel species with $\leqslant 30 \%$ DNA-DNA similarity to its closest phylogenetic neighbours. Vibrio pacinii sp. nov. is proposed, with LMG $19999^{\top}$ (=CAIM $530^{\top}=$ STD3- $1057^{\top}$; DNA G $+C$ content, 44.9 mol\%) as the type strain.
\end{abstract}

Vibrio is an important genus in the culture of marine and estuarine animals, in that many species have been responsible for causing disease in fish (Hjeltnes \& Roberts, 1993), crustaceans (Lightner, 1993) and molluscs (Austin, 1988). Some Vibrio species may also have a beneficial effect in host organisms, either as symbionts [e.g. of squids (McFall-Ngai, 2002)] or probionts (Gomez-Gil et al., 2000). Several studies have investigated phenotypic diversity of vibrios in aquaculture environments (Vandenberghe et al., 1998, 2003). Recently, genotypic techniques have also been employed to better characterize the diversity of vibrios: Thompson et al. (2001) characterized 506 strains by using fluorescent amplified fragment length polymorphism (FAFLP) analysis and found many strains that did not cluster with any known type strains and might thus be considered as potential novel species of the genus Vibrio. This analysis disclosed a group (A47) of three tightly related strains (LMG 13245, LMG $19999^{\mathrm{T}}$ and LMG 21514). These strains were grouped apart from known type strains of Vibrio species, with $>70 \%$ FAFLP pattern similarity between them but only $52 \%$ similarity to that of their closest FAFLP neighbour, strain LMG 10953 (FAFLP group A1). Phenotypic analysis of 1476 Vibrio isolates with the Biolog GN system clustered strains LMG $19999^{\mathrm{T}}$ and LMG 13245 in a separate group (STD3-1057) that was related to Vibrio logei, and strain LMG 21514 clustered in the Vibrio ordalii

Abbreviations: FAFLP, fluorescent amplified fragment length polymorphism; FAME, fatty acid methyl ester.

The GenBank/EMBL/DDBJ accession number for the $16 \mathrm{~S}$ rDNA sequence of strain LMG $19999^{\top}$ is AJ316194. group (Vandenberghe et al., 2003). Austin et al. (1995) reported that strain LMG 13245 had unique ribotype and lipopolysaccharide profiles and no plasmid content, belonged to an unknown serogroup and was pathogenic for Atlantic salmon (Salmo salar). Phenotypic and genotypic methodologies clearly show that bacterioflora, particularly vibrios, found in aquaculture environments represent a highly diverse group with many potential novel species that are yet to be detected and described.

Strain LMG $19999^{\mathrm{T}}\left(=\right.$ CAIM $\left.530^{\mathrm{T}}=\mathrm{STD} 3-1057^{\mathrm{T}}\right)$ was isolated from healthy shrimp larvae (Penaeus chinensis) in the Dahua hatchery in Laizhou (Shandong Province, China) during the spring of 1996 (Vandenberghe et al., 1998). Strain LMG 13245 (=CAIM 526=VIB 218) was isolated from sea bass (Dicentrarchus labrax) in Spain 1991, while strain LMG 21514 (=CAIM 466=VIB 847) was isolated from Atlantic salmon (Salmo salar) in Tasmania during the 1990s. All strains have been deposited at the BCCM/LMG bacteria collection and at the CAIM (Collection of Aquacultural Important Microorganisms, CIAD, A. C. Mazatlán, México).

Strains were grown aerobically on tryptone soy agar (TSA; Oxoid) supplemented with $2 \%(\mathrm{w} / \mathrm{v}) \mathrm{NaCl}$ for $24 \mathrm{~h}$ at $28{ }^{\circ} \mathrm{C}$ unless otherwise stated. Phenotypic characterization was done with the API $20 \mathrm{E}$ system (bioMérieux) to determine the biochemical and nutritional properties of the strains tested, the API ZYM system (bioMérieux) for evaluation of enzymes produced by the strains and the Biolog GN2 system to test the strains' ability to utilize 
different carbon sources. All systems were used according to the manufacturers' instructions, but sterile saline solution $[1.5 \%(\mathrm{w} / \mathrm{v}) \mathrm{NaCl}]$ was used to prepare the inocula in every case. Additional phenotypic tests and confirmation of doubtful test results obtained by these systems were performed by following the methodologies of Lanyi (1987) and Austin \& Lee (1992). Antibiotic sensitivity was estimated by the disc-diffusion test (Bauer et al., 1966) on Iso-Sensitest agar (Oxoid) with $1.5 \%(\mathrm{w} / \mathrm{v}) \mathrm{NaCl}$. GLC analysis of methylated fatty acids was performed as described by Osterhout et al. (1991), but the cells were grown on TSA (Difco) with $1.5 \%(\mathrm{w} / \mathrm{v}) \mathrm{NaCl}$ and incubated at $28^{\circ} \mathrm{C}$. Determination of $\mathrm{G}+\mathrm{C}$ content of the DNA of strain LMG $19999^{\mathrm{T}}$ was determined according to Mesbah et al. (1989) and modified as described by Logan et al. (2000). Strain LMG $19999^{\mathrm{T}}$ (GenBank/EMBL accession no. AJ316194) was analysed further by sequencing the 16S rDNA as described by Thompson et al. (2001). Sequence similarities were obtained with the JukesCantor model [gamma $0 \cdot 4$, pairwise deletion; standard error (SE) estimated by bootstrap method, 1000 replications and random number seed $=67137$ ] with the MEGA program (version 2.1; Kumar et al., 2001). The LMG 19999 sequence was also compared to the sequences in GenBank (BLASTN; Altschul et al., 1990) and the Ribosomal Database Project (RDP; Maidak et al., 1999). Sequences of the closest species and of isolate LMG $19999^{\mathrm{T}}$ were aligned with ClUSTAL X (version 1.8; Thompson et al., 1997). Tree topology (neighbour-joining; Saitou \& Nei, 1987) and stability of groupings (bootstrap analysis, 1000 replicates) were performed with PHYLO_WIN software (Galtier et al., 1996) with Vibrio cholerae as the outlier. DNA-DNA hybridization was done following the methodology described by Willems et al. (2001) at $39^{\circ} \mathrm{C}$.

The three isolates grew as round, yellow, bright colonies on TCBS (thiosulfate/citrate/bile/sucrose) agar; no swarming or luminescence was observed. The isolates were arginine dihydrolase-positive and lysine and ornithine decarboxylase-negative $(\mathrm{A}+, \mathrm{L}-, \mathrm{O}-) . \mathrm{A}+, \mathrm{L}-, \mathrm{O}-$ vibrios represent a diverse group that includes many species (Alsina \& Blanch, 1994a, b). According to the identification scheme provided by Alsina \& Blanch (1994b), strains LMG $19999^{\mathrm{T}}$, LMG 21514 and LMG 13245 would be identified as Vibrio splendidus II or Vibrio furnissii. All three isolates presented many characters that differentiated them from other $\mathrm{A}+, \mathrm{L}-, \mathrm{O}-$ vibrios, e.g. a positive Voges-Proskauer reaction (except for LMG 13245; positive only in Vibrio anguillarum, Vibrio metschnikovii and Vibrio tapetis); negative for indole production (also negative for Vibrio mytili and $V$. tapetis, variable for $V$. splendidus II); and utilization of amygdalin (also by Vibrio diabolicus) but not of D-mannitol (also negative in Vibrio nereis and V. tapetis) (Table 1). Fatty acid content of the strains varied considerably: 28 were detected in strain LMG 13245, 23 in LMG $19999^{\mathrm{T}}$ and only 10 in LMG 21514 (see species description and Table 2). The fatty acid methyl ester (FAME) profiles are in good agreement with those of representatives of the genus Vibrio (Bertone et al., 1996), although two fatty acids were present in higher concentrations. Fatty acids useful for differentiation are shown in Table 1.

16S rDNA sequence similarities from distance matrix calculation and comparison of LMG $19999^{\mathrm{T}}$ to sequences deposited in GenBank (BLASTN) and the Ribosomal Database Project (RDP) II indicated a close relationship to several Vibrio species (Fig. 1), including V. metschnikovii CIP $69.14^{\mathrm{T}}(d=0 \cdot 028, \mathrm{SE}=0 \cdot 005 ; 97 \cdot 4 \%$ BLASTN; $87 \cdot 0 \%$ RDP, 1360 uniquely occurring oligomers), Vibrio kanaloae LMG $20539^{\mathrm{T}}(d=0 \cdot 029, \mathrm{SE}=0 \cdot 005 ; 96 \cdot 6 \%$ BLASTN $)$, Vibrio pomeroyi LMG $20537^{\mathrm{T}}(d=0 \cdot 029, \quad \mathrm{SE}=0 \cdot 005 ; 96 \cdot 4 \%$ BLASTN), Vibrio aestuarianus ATCC $35048^{\mathrm{T}}(d=0 \cdot 031$, $\mathrm{SE}=0 \cdot 005 ; 97 \cdot 7 \%$ BLASTN; $87 \cdot 7 \%$ RDP, 1345 uniquely occurring oligomers), $V$. tapetis CECT $4600^{\mathrm{T}}(d=0 \cdot 033$, $\mathrm{SE}=0 \cdot 005 ; 97 \cdot 3 \%$ BLASTN; $87 \cdot 2 \%$ RDP, 1387 uniquely occurring oligomers) and $V$. furnissii ATCC $35016^{\mathrm{T}}$ $(d=0 \cdot 035, \mathrm{SE}=0 \cdot 005 ; 96 \cdot 6 \%$ BLASTN; $87 \cdot 2 \% \mathrm{RDP}, 1453$ uniquely occurring oligomers). $16 \mathrm{~S}$ rDNA sequence distance within the Vibrio genus for the isolates was 0.028-0.091.

DNA-DNA hybridization experiments revealed high similarity between pairs LMG $19999^{\mathrm{T}}$ and LMG 21514 (79\%), LMG $19999^{\mathrm{T}}$ and LMG 13245 (92\%) and LMG 21514 and LMG $13245(85 \%)$. Similarity of strain LMG $19999^{\mathrm{T}}$ to its closest phylogenetic neighbours was, in decreasing order, $30 \%$ (28\% reciprocal value) with $V$. aestuarianus LMG $7909^{\mathrm{T}}, 28 \%$ (24\% reciprocal value) with $V$. kanaloae LMG $20539^{\mathrm{T}}, 28 \%$ (17\% reciprocal value) with $V$. pomeroyi LMG $20537^{\mathrm{T}}, 21 \%$ with $V$. furnissii LMG $7910^{\mathrm{T}}, 18 \%$ $\left(21 \cdot 8 \%\right.$ reciprocal value) with $V$. metschnikovii LMG $11664^{\mathrm{T}}$ and $16 \%$ (17\% reciprocal value) with $V$. tapetis $\mathrm{LMG}$ $19706^{\mathrm{T}}$.

Collectively, phenotypic characterization, $16 \mathrm{~S}$ rDNA sequences, DNA-DNA similarity and FAFLP fingerprinting (Thompson et al., 2001) provide solid evidence to support the proposal of strains LMG $19999^{\mathrm{T}}$, LMG 21514 and LMG 13245 as members of a novel species of the genus Vibrio, for which the name Vibrio pacinii is proposed.

\section{Description of Vibrio pacinii sp. nov.}

Vibrio pacinii (pa.ci'ni.i. N.L. gen. n. pacinii of Pacini, named after the Italian anatomist Filipo Pacini, who first discovered the causal agent of cholera).

Gram-negative rods, motile, with polar flagella. Nonluminescent, non-pigmented, translucent colonies on marine agar with no swarming. Round, firm, yellow bright colonies on TCBS agar, $1 \cdot 5-2 \cdot 8 \mathrm{~mm}$ in diameter. Grows in the presence of $1.5,2 \cdot 5,6 \cdot 0$ and $8.0 \% \mathrm{NaCl}$ but not at $0,10 \cdot 0$ or $12 \cdot 0 \%$; can grow at 4,30 and $35^{\circ} \mathrm{C}$ but not at $40^{\circ} \mathrm{C}$ in TSB. Oxidase- and catalase-positive, ferments glucose and lactose; arginine and L-tyrosine dihydrolasepositive, lysine and ornithine decarboxylase-negative. Positive for Voges-Proskauer test (except for strain LMG 13245), methyl red, tryptophan deaminase and nitrate 
Table 1. Phenotypic characteristics that differentiate Vibrio pacinii sp. nov. from its closest neighbours and from other arginine dihydrolase-positive, lysine and ornithine decarboxylase-negative Vibrio species

Taxa: 1, V. pacinii; 2, V. kanaloae; 3, V. pomeroyi; 4, V. aestuarianus; 5, V. anguillarum; 6, V. diabolicus; 7, Vibrio diazotrophicus; 8, Vibrio fluvialis; 9, V. furnissii; 10, Vibrio mediterranei; 11, V. metschnikovii; 12, V. mytili; 13, V. nereis; 14, Vibrio orientalis; 15, V. splendidus I; 16, V. splendidus II; 17, V. tapetis; 18, V. tubiashii. Data were obtained from Pujalte et al. (1993), Raguénès et al. (1997), Novoa et al. (1998), Alsina \& Blanch (1994a, b), Farmer (1992), Thompson et al. (2003) and Baumann \& Schubert (1983). +, Positive; -, negative; $\mathrm{V}$, variable; ND, no data available.

\begin{tabular}{|c|c|c|c|c|c|c|c|c|c|c|c|c|c|c|c|c|c|c|}
\hline Test & 1 & 2 & 3 & 4 & 5 & 6 & 7 & 8 & 9 & 10 & 11 & 12 & 13 & 14 & 15 & 16 & 17 & 18 \\
\hline Arginine dihydrolase & + & + & + & + & + & - & $\mathrm{V}$ & + & + & $\mathrm{V}$ & $\mathrm{V}$ & + & + & $\mathrm{V}$ & $\mathrm{V}$ & $\mathrm{V}$ & - & $\mathrm{V}$ \\
\hline Lysine decarboxylase & - & - & - & $\mathrm{V}$ & - & + & - & - & - & $\mathrm{V}$ & $\mathrm{V}$ & - & - & + & - & - & - & - \\
\hline Oxidase & + & + & + & + & + & + & + & + & + & + & - & + & + & + & + & + & ND & + \\
\hline Resistance to $\mathrm{O} / 129(10 \mu \mathrm{g})$ & - & - & - & - & + & ND & $\mathrm{V}$ & + & + & - & - & ND & $\mathrm{V}$ & - & - & $\mathrm{V}$ & - & - \\
\hline ONPG & $\mathrm{V}$ & - & + & + & + & - & + & $\mathrm{V}$ & + & + & + & + & - & + & $\mathrm{V}$ & $\mathrm{V}$ & + & + \\
\hline Citrate & + & - & - & + & + & + & + & + & + & - & - & + & + & + & + & + & - & + \\
\hline Nitrate reduction & + & + & + & + & + & + & + & + & + & + & - & + & + & + & + & + & + & + \\
\hline Gelatinase & $\mathrm{V}$ & + & + & + & + & + & - & + & V & - & + & - & $\mathrm{V}$ & + & + & + & + & + \\
\hline Urease & - & - & - & - & - & - & + & - & - & - & - & - & - & - & - & - & - & $\mathrm{V}$ \\
\hline $8 \% \mathrm{NaCl}$ & + & $\mathrm{V}$ & + & $\mathrm{V}$ & - & $\mathrm{ND}$ & + & $\mathrm{V}$ & + & $\mathrm{V}$ & $\mathrm{V}$ & + & + & + & $\mathrm{V}$ & $\mathrm{V}$ & - & $\mathrm{V}$ \\
\hline $10 \% \mathrm{NaCl}$ & - & - & - & - & + & $\mathrm{ND}$ & $\mathrm{V}$ & $\mathrm{V}$ & + & - & $\mathrm{V}$ & + & $\mathrm{V}$ & - & - & - & - & - \\
\hline $4^{\circ} \mathrm{C}$ & + & + & + & + & - & + & V & - & - & - & - & - & $\mathrm{V}$ & + & V & - & + & - \\
\hline $35^{\circ} \mathrm{C}$ & $\mathrm{V}$ & - & - & + & + & + & + & + & + & $\mathrm{V}$ & + & + & + & + & V & - & - & $\mathrm{V}$ \\
\hline $40^{\circ} \mathrm{C}$ & - & - & - & - & - & + & $\mathrm{V}$ & + & + & - & + & - & $\mathrm{V}$ & - & - & - & $\mathrm{ND}$ & - \\
\hline \multicolumn{19}{|l|}{ Utilization of: } \\
\hline D-Mannitol & - & + & + & + & + & + & + & + & + & + & + & + & - & + & + & + & - & + \\
\hline D-Mannose & + & + & + & + & + & + & - & + & $\mathrm{V}$ & + & + & - & - & + & + & - & - & + \\
\hline myo-Inositol & - & - & - & - & $\mathrm{V}$ & - & - & - & - & + & + & - & - & - & - & - & - & - \\
\hline D-Sorbitol & - & - & - & + & + & - & - & - & - & + & - & - & - & - & - & - & - & - \\
\hline
\end{tabular}

reduction; negative for indole production, $\mathrm{H}_{2} \mathrm{~S}$ and urease. Test for citrate utilization is weakly positive, except for strain LMG 13245, which is strongly positive. Positive activity of acid phosphatase (LMG 13245 is weak), $\alpha$-glucosidase (LMG 21514 is weak), alkaline phosphatase, esterase (C4), esterase lipase (C8) (LMG 13245 is weak), leucine arylamidase, naphthol-AS-BI-phosphohydrolase and valine arylamidase (LMG 13245 and LMG 21514 are weak). Negative activity of $\alpha$-chymotrypsin (LMG $19999^{\mathrm{T}}$ is weakly positive), $\alpha$-fucosidase, $\alpha$-galactosidase, $\alpha$-mannosidase, $\beta$-glucosidase, $\beta$-glucuronidase, lipase (C14), $N$-acetyl- $\beta$-glucosaminidase and trypsin. All strains utilize $\alpha$-D-glucose, cellobiose, dextrin, D-fructose, D-mannitol, D-trehalose, gentiobiose, inosine, L-asparagine,
L-glutamic acid, L-serine, maltose, $\mathrm{N}$-acetyl-D-glucosamine, sucrose, thymidine and uridine as sole sources of carbon. None of the strains utilized 2,3-butanediol, 2-aminoethanol, acetic acid, adonitol, alaninamide, $\alpha$-D-lactose lactulose, $\alpha$-hydroxybutyric acid, $\alpha$-ketobutyric acid, $\alpha$-ketoglutaric acid, $\alpha$-ketovaleric acid, $\alpha$-lactose, $\beta$-hydroxybutyric acid, bromosuccinic acid, cis-aconitic acid, citric acid, DL- $\alpha$-glycerol phosphate, DL-carnitine, D-alanine, Darabitol, D-galactonic acid lactone, D-galacturonic acid, D-gluconic acid, D-glucosaminic acid, D-glucuronic acid, D-mannose, D-melibiose, D-raffinose, D-saccharic acid, Dserine, formic acid, $\gamma$-aminobutyric acid, $\gamma$-hydroxybutyric acid, glucose 1-phosphate, glucose 6-phosphate, glucuronamide, glycerol, glycyl L-glutamic acid, hydroxy 
Table 2. Phenotypic differences between strains of $V$. pacinii sp. nov.

Strains: 1, LMG 19999 ${ }^{\mathrm{T}}$; 2, LMG 21514; 3, LMG 13245. +, Positive; -, negative; W, weak result; S, sensitive; I, intermediate; $\mathrm{R}$, resistant; ND, not detected.

\begin{tabular}{|c|c|c|c|}
\hline Test & 1 & 2 & 3 \\
\hline Voges-Proskauer reaction & + & + & - \\
\hline \multicolumn{4}{|l|}{ Utilization of: } \\
\hline$\alpha$-Cyclodextrin & - & - & + \\
\hline Citrate & $\mathrm{W}$ & $\mathrm{W}$ & + \\
\hline Methyl $\beta$-D-glucoside & + & - & - \\
\hline DL-Lactic acid & + & - & + \\
\hline D-Galactose & + & - & + \\
\hline D-Sorbitol & - & - & + \\
\hline Glycogen & - & - & + \\
\hline Glycyl L-aspartic acid & $\mathrm{w}$ & + & - \\
\hline L-Alanine & + & $\mathrm{W}$ & + \\
\hline L-Alanylglycine & + & $\mathrm{W}$ & + \\
\hline L-Aspartic acid & + & + & - \\
\hline Methyl pyruvate & + & - & - \\
\hline Monomethyl succinate & - & + & - \\
\hline Psicose & + & - & + \\
\hline Succinic acid & + & + & - \\
\hline \multicolumn{4}{|l|}{ Activity of: } \\
\hline$\beta$-Galactosidase & + & - & + \\
\hline Cystine arylamidase & + & $\mathrm{w}$ & - \\
\hline \multicolumn{4}{|l|}{ Fatty acid content (\%): } \\
\hline iso- $\mathrm{C}_{16: 0}$ & $8 \cdot 41$ & ND & $9 \cdot 69$ \\
\hline iso- $\mathrm{C}_{14: 0} 3-\mathrm{OH}$ & $2 \cdot 71$ & ND & $1 \cdot 08$ \\
\hline $\mathrm{C}_{16: 0}$ & $14 \cdot 07$ & $25 \cdot 55$ & $16 \cdot 62$ \\
\hline \multicolumn{4}{|l|}{ Susceptibility to: } \\
\hline Ampicillin $(30 \mu \mathrm{g})$ & S & S & $\mathrm{R}$ \\
\hline Amikacin $(30 \mu \mathrm{g})$ & I & S & $\mathrm{R}$ \\
\hline
\end{tabular}

L-proline, i-erythritol, itaconic acid, L-arabinose, L-fucose, L-histidine, L-leucine, L-ornithine, L-phenylalanine, Lproline, L-pyroglutamic acid, L-rhamnose, L-threonine, malonic acid, $m$-inositol, $N$-acetyl-D-galactosamine, phenylethylamine, $p$-hydroxyphenylacetic acid, propionic acid, putrescine, quinic acid, sebacic acid, succinamic acid, turanose, Tween 40, Tween 80 , urocanic acid or xylitol. The following cellular fatty acids are present in decreasing order (mean percentage of the three strains analysed, minimum and maximum of total fatty acid content): $\mathrm{C}_{16: 0}(18 \cdot 7 \%$, $14 \cdot 1-25 \cdot 5), \mathrm{C}_{18: 1} \omega 7 c(11 \cdot 1 \%, 9 \cdot 9-13 \cdot 4)$, iso- $\mathrm{C}_{16: 0}(9 \cdot 0 \%$, 8.4-9.7, not detected in LMG 21514), $\mathrm{C}_{14: 0}(6 \cdot 9 \%$, $4 \cdot 7-11 \cdot 2), \mathrm{C}_{12: 0}(3 \cdot 7 \%, 1 \cdot 9-5 \cdot 2), \mathrm{C}_{12: 0} 3-\mathrm{OH}(3 \cdot 1 \%$, $1 \cdot 5-4 \cdot 9)$, iso- $\mathrm{C}_{14: 0} 3-\mathrm{OH}(1 \cdot 9 \%, 1 \cdot 1-2 \cdot 7$, not detected in LMG 21514), iso- $\mathrm{C}_{17: 0}(1 \cdot 4 \%, 0 \cdot 9-2 \cdot 0$, not detected in LMG 21514), $\mathrm{C}_{16: 1} \omega 7 c$ and/or iso- $\mathrm{C}_{15: 0}$ 2-OH (summed feature 3$)(38 \cdot 7 \%, 37 \cdot 8-39 \cdot 2)$ and $\mathrm{C}_{14: 0} 3-\mathrm{OH}$ and/or iso$\mathrm{C}_{16: 1} \mathrm{I}$ (summed feature 2) $(2 \cdot 9 \%, 1 \cdot 5-3 \cdot 6)$. Eighteen other fatty acids are present in percentages below $1 \cdot 0$, these are: iso- $\mathrm{C}_{18: 0}$, iso- $\mathrm{C}_{14: 0}, \mathrm{C}_{16: 1} \omega 7 c$ alcohol, iso- $\mathrm{C}_{13: 0}$, iso- $\mathrm{C}_{15: 0}$, anteiso- $\mathrm{C}_{17: 0}, \mathrm{C}_{18: 0}, \mathrm{C}_{15: 0}, \mathrm{C}_{17: 0}, 11$-methyl $\mathrm{C}_{18: 1} \omega 7 c$,

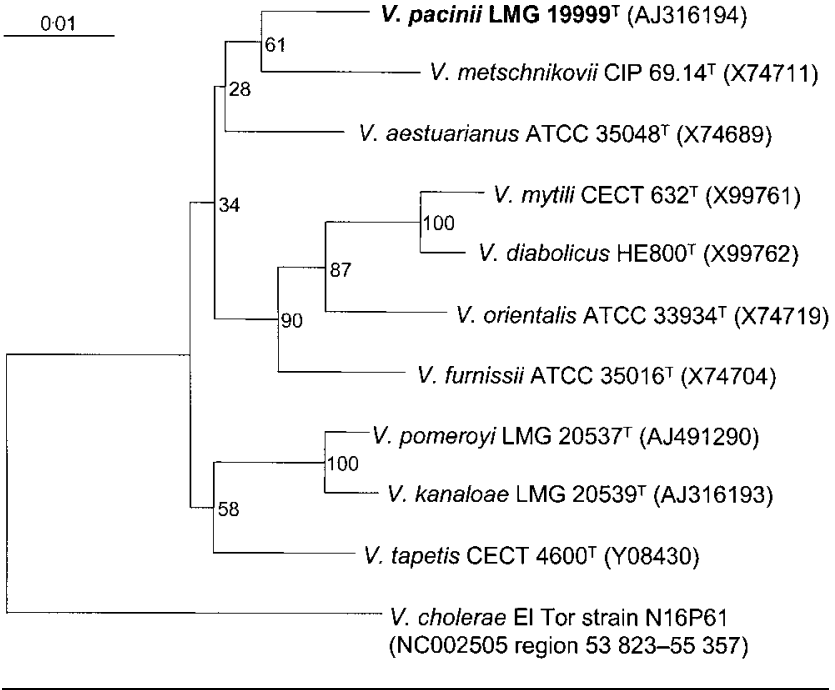

Fig. 1. Consensus phylogenetic dendrogram of strain LMG $19999^{\top}\left(=\right.$ CAIM 530 ${ }^{\top}$ ) and the closest Vibrio species, derived from almost-complete $16 \mathrm{~S}$ rDNA sequence data. Tree topology was obtained by neighbour-joining $(0.4 \gamma$-correction, pairwise deletion, Jukes-Cantor correction). Numbers at nodes indicate level of bootstrap support (1000 replicates). Bar, $1 \%$ sequence divergence.

$\mathrm{C}_{17: 1} \omega 8 c$, iso- $\mathrm{C}_{13: 0} 3-\mathrm{OH}$, iso- $\mathrm{C}_{15: 0} 3-\mathrm{OH}$, iso- $\mathrm{C}_{12: 0}, \mathrm{C}_{12: 0}$ 2-OH, anteiso- $\mathrm{C}_{15: 0}, \mathrm{C}_{15: 1} \omega 8 c$ and $\mathrm{C}_{16: 1} \omega 5 c$. Antibiotic susceptibility was observed to chloramphenicol $(30 \mu \mathrm{g})$, oxolinic acid $(2 \mu \mathrm{g})$, oxytetracycline $(30 \mu \mathrm{g})$, polymyxin $\mathrm{B}$ $(300 \mathrm{U})$, tetracycline $(30 \mu \mathrm{g})$; resistant to gentamicin $(10 \mu \mathrm{g})$ (except for LMG 21514, which is intermediate), kanamycin $(30 \mu \mathrm{g})$, streptomycin $(25 \mu \mathrm{g})$ and vibriostatic agent $\mathrm{O} / 129$ at 10 and $150 \mu \mathrm{g}$. Additional phenotypic features are listed in Table $2 . \mathrm{G}+\mathrm{C}$ content of the DNA is $44 \cdot 9 \mathrm{~mol} \%$.

The type strain, LMG $19999^{\mathrm{T}}$ (=CAIM $530^{\mathrm{T}}$ ), was isolated from shrimp larvae (Penaeus chinensis) in the Dahua hatchery in Laizhou (Shandong Province, China). Reference strains are LMG 13245 (=CAIM 526) and LMG 21514 ( = CAIM 466).

\section{Acknowledgements}

This study was financed by CONACyT (México) project J-28344 to B. G., Conselho Nacional de Desenvolvimento Científico e Tecnológico (CNPq, Brazil) as PhD scholarship no. 2008361/98-6 for F. L. T. and Fund for Scientific Research FWO (Belgium) grants to J. S. Thanks to Cipatli Meza C. and Carmen Bolan M.

\section{References}

Alsina, M. \& Blanch, A. R. (1994a). A set of keys for biochemical identification of environmental Vibrio species. J Appl Bacteriol 76, $79-85$. 
Alsina, M. \& Blanch, A. R. (1994b). Improvement and update of a set of keys for biochemical identification of Vibrio species. J Appl Bacteriol 77, 719-721.

Altschul, S. F., Gish, W., Miller, W., Myers, E. W. \& Lipman, D. J. (1990). Basic local alignment search tool. J Mol Biol 215, 403-410.

Austin, B. (1988). Marine Microbiology. Cambridge: Cambridge University Press.

Austin, B. \& Lee, J. V. (1992). Aeromonadaceae and Vibrionaceae. In Identification Methods in Applied and Environmental Microbiology, pp. 163-182. Edited by R. G. Board, D. Jones \& F. A. Skinner. Oxford: Blackwell Scientific Publications.

Austin, B., Alsina, M., Austin, D. A. \& 9 other authors (1995). Identification and typing of Vibrio anguillarum: a comparison of different methods. Syst Appl Microbiol 18, 285-302.

Bauer, A. W., Kirby, W. M., Sherris, J. C. \& Turck, M. (1966). Antibiotic susceptibility testing by a standardized single disk method. Am J Clin Pathol 45, 493-496.

Baumann, P. \& Schubert, R. H. W. (1983). Vibrionaceae. In Bergey's Manual of Systematic Bacteriology, vol. 1, pp. 516-550. Edited by N. R. Krieg \& J. G. Holt. Baltimore: Williams \& Wilkins.

Bertone, S., Giacomini, M., Ruggiero, C., Piccarolo, C. \& Calegari, L. (1996). Automated systems for identification of heterotrophic marine bacteria on the basis of their fatty acid composition. Appl Environ Microbiol 62, 2122-2132.

Farmer, J. J. (1992). The family Vibrionaceae. In The Prokaryotes. A Handbook on the Biology of Bacteria: Ecophysiology, Isolation, Identification, Applications, pp. 2938-2951. Edited by A. Balows. New York: Springer-Verlag.

Galtier, N., Gouy, M. \& Gautier, C. (1996). SEAVIEW and PHYLO_WIN: two graphic tools for sequence alignment and molecular phylogeny. Comput Appl Biosci 12, 543-548.

Gomez-Gil, B., Roque, A. \& Turnbull, J. F. (2000). The use and selection of probiotic bacteria for use in the culture of larval aquatic organisms. Aquaculture 191, 259-270.

Hjeltnes, B. \& Roberts, R. J. (1993). Vibriosis. In Bacterial Diseases of Fish, pp. 109-121. Edited by V. Inglis, R. J. Roberts \& N. R. Bromage. Oxford: Blackwell Scientific Publications.

Kumar, S., Tamura, K., Jakobsen, I. B. \& Nei, M. (2001). MEGA2: molecular evolutionary genetic analysis software. Bioinformatics $\mathbf{1 7}$, 1244-1245.

Lanyi, B. (1987). Classical and rapid identification methods for medically important bacteria. In Current Methods for Classification and Identification of Microorganisms, pp. 1-67. Edited by R. R. Colwell \& R. Grigorova. London: Academic Press.

Lightner, D. V. (1993). Diseases of cultured penaeid shrimp. In $C R C$ Handbook of Mariculture: Crustacean Aquaculture, 2nd edn, vol. 1, pp. 393-486. Edited by J. P. McVey. Boca Raton, FL: CRC Press.

Logan, N. A., Lebbe, L., Hoste, B. \& 7 other authors (2000). Aerobic endospore-forming bacteria from geothermal environments in northern Victoria Land, Antarctica, and Candlemas Island, South
Sandwich archipelago, with the proposal of Bacillus fumarioli sp. nov. Int J Syst Evol Microbiol 50, 1741-1753.

Maidak, B. L., Cole, J. R., Parker, C. T., Jr \& 11 other authors (1999). A new version of the RDP (Ribosomal Database Project). Nucleic Acids Res 27, 171-173.

McFall-Ngai, M. J. (2002). Unseen forces: the influence of bacteria on animal development. Dev Biol 242, 1-14.

Mesbah, M., Premachandran, U. \& Whitman, W. B. (1989). Precise measurement of the $\mathrm{G}+\mathrm{C}$ content of deoxyribonucleic acid by highperformance liquid chromatography. Int J Syst Bacteriol 39, 159-167.

Novoa, B., Luque, A., Castro, D., Borrego, J. J. \& Figueras, A. (1998). Characterization and infectivity of four bacterial strains isolated from brown ring disease-affected clams. J Invertebr Pathol 71, 34-41.

Osterhout, G. J., Shull, V. H. \& Dick, J. D. (1991). Identification of clinical isolates of gram-negative nonfermentative bacteria by an automated cellular fatty acid identification system. J Clin Microbiol 29, 1822-1830.

Pujalte, M.-J., Ortigosa, M., Urdaci, M.-C., Garay, E. \& Grimont, P. A. D. (1993). Vibrio mytili sp. nov., from mussels. Int J Syst Bacteriol 43, $358-362$.

Raguénès, G., Christen, R., Guezennec, J., Pignet, P. \& Barbier, G. (1997). Vibrio diabolicus sp. nov., a new polysaccharide-secreting organism isolated from a deep-sea hydrothermal vent polychaete annelid, Alvinella pompejana. Int J Syst Bacteriol 47, 989-995.

Saitou, N. \& Nei, M. (1987). The neighbor-joining method: a new method for reconstructing phylogenetic trees. Mol Biol Evol 4, 406425.

Thompson, J. D., Gibson, T. J., Plewniak, F., Jeanmougin, F. \& Higgins, D. G. (1997). The CLUSTAL_X windows interface: flexible strategies for multiple sequence alignment aided by quality analysis tools. Nucleic Acids Res 25, 4876-4882.

Thompson, F. L., Hoste, B., Vandemeulebroecke, K. \& Swings, J. (2001). Genomic diversity amongst Vibrio isolates from different sources determined by fluorescent amplified fragment length polymorphism. Syst Appl Microbiol 24, 520-538.

Thompson, F. L., Thompson, C. C., Li, Y., Gomez-Gil, B., Vandenberghe, J., Hoste, B. \& Swings, J. (2003). Vibrio kanaloae sp. nov, Vibrio pomeroyi sp. nov. and Vibrio chagasii sp. nov., from sea water and marine animals. Int J Syst Evol Microbiol 53, 753-759.

Vandenberghe, J., Li, Y., Verdonck, L., Li, J., Sorgeloos, P., Xu, H. S. \& Swings, J. (1998). Vibrios associated with Penaeus chinensis (Crustacea: Decapoda) larvae in Chinese shrimp hatcheries. Aquaculture 169, 121-132.

Vandenberghe, J., Thompson, F. L., Gomez-Gil, B. \& Swings, J. (2003). Phenotypic diversity amongst Vibrio isolates from marine aquaculture systems. Aquaculture 219, 9-20.

Willems, A., Doignon-Bourcier, F., Goris, J., Coopman, R., de Lajudie, P., De Vos, P. \& Gillis, M. (2001). DNA-DNA hybridization study of Bradyrhizobium strains. Int J Syst Evol Microbiol 51, 1315-1322. 